\title{
Research Paper: Objective Peak-Detection in Complex Auditory Brainstem Response to /ba/, /da/, /ga/: A Novel Technique
}

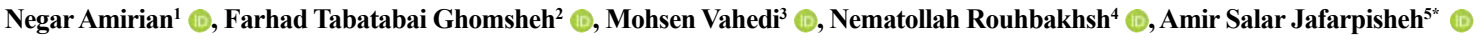 \\ 1. Department of Biomedical Engineering, Faculty of Technology and Engineering, Central Tehran Branch, Islamic Azad University, Tehran, Iran. \\ 2. Pediatric Neurorehabilitation Research Center, University of Social Welfare and Rehabilitation Sciences, Tehran, Iran. \\ 3. Department of Biostatistics, University of Social Welfare and Rehabilitation Sciences, Tehran, Iran. \\ 4. Department of Audiology, School of Rehabilitation, Tehran University of Medical Sciences, Tehran, Iran. \\ 5. Department of Ergonomics, University of Social Welfare and Rehabilitation Sciences, Tehran, Iran.
}

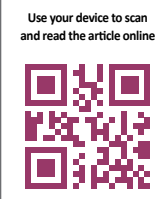

Citation: Amirian N, Tabatabai Ghomsheh F, Vahedi M, Rouhbakhsh N, Jafarpisheh AS. Objective Peak-Detection in Complex Auditory Brainstem Response to /ba/, /da/, /ga/: A Novel Technique. Iranian Rehabilitation Journal. 2018; 16(3):219-232. http://dx.doi.org/10.32598/irj.16.3.219

: http://dx.doi.org/10.32598/irj.16.3.219

\section{(c) (1) (8)}

Funding: See Page 230

Article info:

Received: 10 Jan 2018

Accepted: 25 May 2018

Available Online: 01 Sep 2018

\section{Keywords:}

Auditory evoked potentials, Stimulus-specific adaptation, Linguistic effect, Speech perception, Hearing loss, Auto peak detection

\section{ABSTRACT}

Objectives: The result of auditory brainstem response is used worldwide for detecting hearing impairments or hearing aids. This study aimed to introduce the superiority of mathematical innovation algorithm toward subjective evaluation by an audiologist. The automatic algorithm method is encouraged for detecting the waves of Auditory Brainstem Response (ABR), because it can reduce subjective evaluation biases and visual analysis errors. This article portrays another technique for automatic detection of the peaks. Finally, by obtaining the standard pattern with this automatic algorithm for Persian speakers, we will compare it with the English speakers whose information was obtained by subjective method in Northwestern University. This article describes the effect of different factors on brainstem responses by performing a new automatic method.

Methods: Auditory evoked potentials of brainstem activity were recorded by Electro encephalogram (EEG) of 27 Persian speaker adults with normal hearing. Three stimulus /ga/, / $\mathrm{da} /$, and $/ \mathrm{ba} /$ were presented. This strategy depends on the utilization of reference wave forms, time latencies, and peaks adjusted and comparison with the ABR. Brainstem response latencies of brainstem peaks were extracted by the automatic method in temporal and spectral domains. This step provides language patterns for Persian speakers. Finally, the results of Persian speakers were compared with the results of a previous study done in Northwestern University by the same recording protocol as our own study on 22 English speaker children. Intraclass correlation coefficients and paired $t$ test were used for evaluating and comparing the results.

Results: According to the results, the performance of automatic method is high and reliable. Automatic and visual analysis methods had significant interaction. Latency of auditory brainstem response to the same stimulus in the two study groups was different and had a significant latency. The significance of these discoveries and clinical outcomes of this target strategy are featured in this paper.

Discussion: This simple innovative algorithm could find the correct location of ABR peaks. Because of different acoustic signs and symptoms in the brainstem, the time latencies for all three stimulus used in this study are completely different.

\footnotetext{
* Corresponding Author:

Amir Salar Jafarpisheh, PhD

Address: Department of Ergonomics, University of Social Welfare and Rehabilitation Sciences, Tehran, Iran.

Tel: +98 (21) 22180119

E-mail:am.jafarpisheh@uswr.ac.ir
} 


\section{Highlights}

- Automatic central auditory brainstem response (cABR) peak detection is possible.

- Responses to different stimuluses have different time scale patterns.

- There is a significant correlation between automatic and experts peak detection method.

\section{Plain Language Summary}

The Auditory Brainstem Response (ABR) is used worldwide for hearing screening purposes. This study is about complex auditory brainstem responses to /da/, /ba/, /ga/. This assessment is normally done manually by an audiologist. The utilization of programmed techniques for peak detection can improve procedure performance. Latency is an important factor for evaluating the activity of neural networks in brainstem for each stimuli. Using automatic peak detection algorithm instead of visual peak marking could reduce errors and increase precision of the analysis. Based on marked peaks with experts, automatic peak detection algorithm was developed and examined.

\section{Introduction}

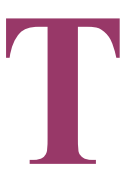

he Auditory Brainstem Response (ABR) is the electrical activity of the auditory nerve generated in the brainstem. It is recorded by electrodes of non-invasive tools such as EEG. ABR is associated with similar response to non-verbal and verbal stimuli $[1,2]$. The use of verbal stimuli is preferable to other stimuli as verbal or speech stimuli provide biological processes underpinning normal auditory processing and auditory processing disorders [2].

ABRs are represented in temporal and spectral phases of stimulus by different neural population in different regions of the brainstem. The signals of ABRs are classified in two parts; non-periodic brief stimuli evoked by transient responses and periodic long-term stimuli evoked by sustained responses [2,3]. More specifically, speech or the complex Auditory Brainstem Response (cABR) can preserve pitch, formants, and the timing of speech stimuli, crucial in speech understanding both in calm and within the sight of foundation commotion [1-3].

Different factors such as different languages according to each racial, music or speech experience, the period of auditory training (short-term or long-term) and hearing loss disorders can influence ABRs [2,3]. These factors shape the morphology of ABR signals and could reflect (induce) the plasticity of the neural networks at the level of brainstem [1]. In addition, differences in age, complex speech stimuli elements and the frequency of stimuli can evoke different responses [3, 4].
Consonant-Vowel (CV) combinations have rich consonant structures, dynamic abundance adjustments, and fast spectrotemporal vacillations due to changes in the filter function of the vocal tracts in source-filter model. Different types of CVs influence neural phase locking and cause changes in latency time. Stimuli with high frequencies has short latency time and process in cochlea, but stimuli with low frequencies has long latency time and process in apical of cochlea $[2,4,5]$.

The source of the human voice is the vocal fold vibration. It vibrates at the fundamental voice pitch and the filter is the function of passages above larynx which shapes the voice coming up from the vocal chords. The source-filter model could be represented by a spectrograph. For example, different synthesized CVs such as $/ \mathrm{ba} /, / \mathrm{da} /$, and $/ \mathrm{ga} /$ create various spectrograms [3]. The duration of CVs vary in phonation length from 40 to $500 \mathrm{~ms}$. The duration of phonation affects pitch and other acoustic features such as harmonics, formants, and formant transition $[1,3,4]$. The formant transition of vowels involve F1, F2, and F3 components and F0 is the fundamental frequency, also the vibration rate of vocal chords could determine the formant transition [1, 3]. The particular segments of the brainstem reaction mirror the acoustic attributes of pitch and formants independently. High frequencies and lower frequencies are responded by basal regions of the cochlea and apical regions, respectively [1].

Different methods of processing were used for analysis and extracting the main ABR waveform elements from ABR signals through detecting major and minor peaks and valleys. Generally, ABR signal analysis is performed 
in temporal and frequency domains $[2,3]$. Extracting features of brainstem response and consonant-vowel stimuli are implemented through the linear and non-linear automatic methods. Using automatic methods could increase the quality of response assessment, peak detection, and improve the quality of the assessment of processing, i.e. automatically stopping averaging sand could ignore recording of unnecessary sweeps in ABRs [6-8].

Numerous automatic methods such as zero crossing method [5, 9], adaptive signal enhancement [5, 10], multi-filters $[5,11]$, single-trial covariance analysis $[5$, $12]$, and automatic peak picking are presented so far $[5,13,14]$. The most common reported strategies of automatic ABR analysis is the correlation coefficient between two consecutive ABR signals $[5,15]$. A subjective evaluation by audiologists is the most common method of analysis for assessing the ABR response. This evaluation may differ from one audiologist to another $[9,14,16,17]$.

Automatic methods could solve representational bias and increase assessment accuracy. Automatic methods of identification promote objective methods. Although a number of objective methods have been developed in automatic evaluation of ABR, a few have been implemented in commercial devices. This article aimed to provide another target strategy for the automatic assessment of the nature of ABR signals and evident proof of the peaks in view of the utilization of templates waves. Also this article examines the synthetic use of preprocessing methods like Signal-to-Noise Ratio (SNR) of the response, correlation coefficient between grand average signal and each stimuli (/da/,/ba/,/ga/) responses signal, and stimuli signal and each response signal of cABR in Persian speakers by MATLAB software.

By using this innovative method, recording unnecessary sweeps and detecting each unnecessary and non-standard peaks are avoided. Finally, thanks to this method, lan- guage patterns of Persian speakers could be extracted and compared with the studies on English speakers [2].

\section{Methods}

This part describes a simple novel objective technique of extracting cABR features such as amplitude, width, latency, as well as automatic detection of peaks.

\section{Participants}

A total of 27 adult (13 female and 14 male) students with the Mean \pm SD age of $24.34 \pm 1.95$ years (age range: 22-29 years) from Tehran University of Medical Sciences participated in this study. All participants were native monolingual speakers of Persian with normal hearing and no neurological disorders. The hearing thresholds of participants were $20 \mathrm{~dB}$ HL or higher at octave frequencies $(250-8000 \mathrm{~Hz})$.

\section{Stimuli and presentation}

Three diotic synthesized consonant-vowel combinations including /da/, /ba/, and/ga/ with $170 \mathrm{~ms}$ duration at the rate of $20 \mathrm{kHz}$ were presented to each person. Stimuli were obtained from the study of Kraus and et al. [2]. Formant transition with duration of $50 \mathrm{~ms}$ and linearly rising included formant number $1(400-720 \mathrm{~Hz})$, flat formant number $4(3300 \mathrm{~Hz})$, formant number $5(3750 \mathrm{~Hz})$, and formant number $6(4900 \mathrm{~Hz})$ (Figure 1). Initial frication were centered at frequencies around formant number 4 (F4) and formant number 5 (F5) in $10 \mathrm{~ms}$.

After $50 \mathrm{~ms}$ of formant transition period, formant number 2 (F2) and formant number 3 (F3) remained consistent at their progression endpoint in $1240 \mathrm{~Hz}$ and 2500 $\mathrm{Hz}$, respectively [2, 18]. The starting point of F2 and F3 were the portions of different stimuli. For [ba], F2 and F3 rose from $900 \mathrm{~Hz}$ and $2400 \mathrm{~Hz}$, respectively. For [da], F2 and F3 tumbled down from 1700 and $2580 \mathrm{~Hz}$, individually. For [ga], F2 and F3 reduced from 3000 and 3100
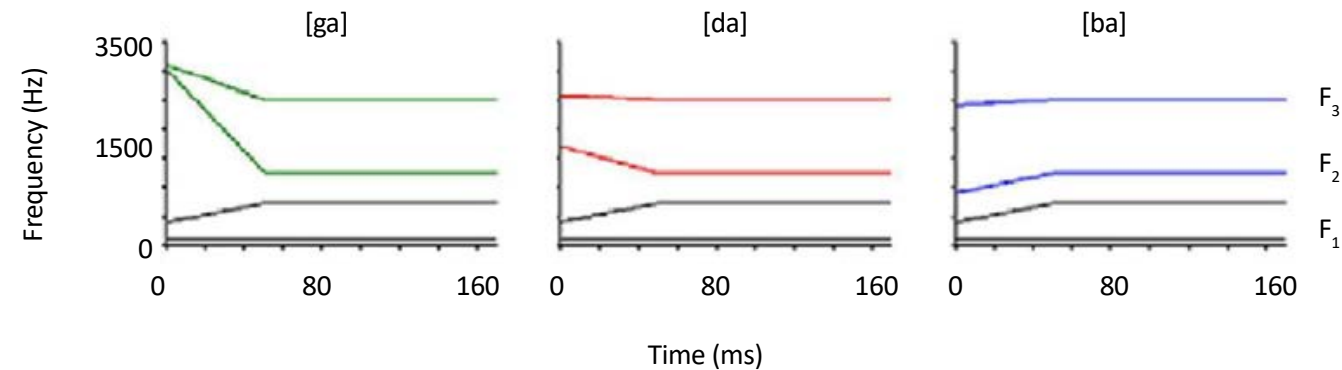

Figure 1. left: /ga/ Schematic, center: /da/ Schematic, right: / ba/ Schematic stimuli in ms [2] 
$\mathrm{Hz}$, individually. These blended boost have an identical and consistent F0 in whole length $[2,18]$.

The details of formant transition related to these three stimuli are presented in Table 1. After passing $50 \mathrm{~ms}$ of formant transition period, F2 and F3 in 1240 to $2500 \mathrm{~Hz}$, respectively remained constant at their transition endpoint frequencies of 1240 and $2500 \mathrm{~Hz}$. The dissimilarities reflected in F2 and F3, for [ba], F2 and F3 rose from $900 \mathrm{~Hz}$ and $2400 \mathrm{~Hz}$. For [da], F2 and F3 descent from 1700 and 2580. For [ga], F2 and F3 decreased from 3000 and 3100 , respectively.

The F0 of these three diotic stimulus were identical and constant in the entire duration $[2,10]$. The diotic stimuli was presented at the rate of $4.65 / \mathrm{s}$, and both stimulus polarities (build-up and rarefaction) were exhibited. The trial of stimulus was introduced to the correct ear through Etymotic's ER-3 headphones (Etymotic Research, Elk Grove Village, IL), at the force of $83 \mathrm{db}$ SPL. Videotaped program were presented to all subjects to promote their cooperation and stillness $[2,10]$.

\section{Recording parameters}

Continuous g.tec EEG was used for recording evoked potentials synchronized with auditory stimuli. Electrodes were located from $\mathrm{Cz}$ to ipsilateral earlobe, with forehead served as ground, band pass filtered from 0.05 $\mathrm{Hz}$ to $3000 \mathrm{~Hz}$, and digitized at $20000 \mathrm{~Hz}$. All electrodes were made with $\mathrm{Ag} / \mathrm{AgCl}$ and their impedance was lower than $5 \mathrm{k} \Omega$. For each stimuli, EEG was processed offline for creating average signals. An EEG was divided into $230 \mathrm{~ms}$ epochs (45 ms pre stimulus onset to $185 \mathrm{~ms}$ post stimulus) and each epoch was band pass filtered from 70 to $2000 \mathrm{~Hz}$ for isolating the brainstem response frequencies.

An artifact criterion of $\pm 35 \mathrm{mV}$ was applied in reject myogenic artifacts. The processed epochs were separately averaged for each stimuli according to their polarity, then they were summed up to isolate the neural response [2, 10, 19]. Final averaged for each stimuli ranged between 4000 to 4100 sweeps per subject for each stimulus [2].

\section{Analysis}

\section{Formant transition period analysis}

The formant transition is a part of response that corresponds to the onset with duration of 0-70 ms. According to different stimuli, latency is altered in this portion.
To isolate formant transition part and eliminate low-frequency activity that could obscure variation of latency, response waveform was additionally high-pass filtered at $300 \mathrm{~Hz}$. First $70 \mathrm{~ms}$ of transition waveform of grand average response was selected for temporal analysis. For Fast Fourier Transform analysis (FFT), 18-58 ms of formant transition average was calculated in $50 \mathrm{~Hz}$ wide bins surrounding F0 and next 10 harmonics.

The range of 400-720 Hz referring to F0 frequency was chosen, then all 10 harmonic peaks marked for each stimulus by MATLAB software via Intraclass Correlation Coefficients (ICC), and Paired t test for evaluating frequency bin difference were performed in each stimulus. ABRs of each subject had 16 peaks in this portion, and peaks 1 , and 2 were the starting point and called onset response. Peaks 3, 5, 6, 7, 9, 10, 12, 13, 15, and 16 were the major peaks, and peaks $5,8,11$, and 14 were the minor peaks. The peaks 15 and 16 were the end point of transient response where the acoustic properties of the three stimulus were identical.

The grand-average signal marked by manual method is shown in Figure 2. The grand average signal for each stimulus with the use of the reference lookup Table is shown in Figure 2.A, 2.B, 2.C. As per the visual analysis report of an audiologist, the onset of response revealed in $9 \mathrm{~ms}$, with every $\sim 10 \mathrm{~ms}$ major peaks of activity occurring around $23 \mathrm{~ms}$. According to this theory, we estimated the grand average of each of three stimuli response and divided it into 7 epochs with duration of 10 $\mathrm{ms}$. Starting epoch of this portion included 2 peaks $(1,2)$ that was called the starting point of response. Also, endpoint included 2 peaks $(15,16)$, called as the offset, but other epochs included 3 peaks (positive, negative major peaks and minor peaks). In this technique, after estimating positive and negative major peaks, maximum and minimum amplitude of each signal were evaluated by calculating $\mathrm{f}(\mathrm{c})$. In this phase, we set limitation periods for identifying direction curvature and detection peaks in each epoch, according to Equation (1).

(1) $f(c)=\left\{\begin{array}{l}y^{\prime}=0 y^{\prime}<0 \\ y^{\prime}=0 y^{\prime}>0\end{array}\right.$

Two audiology experts, professional in speech ABR, marked grand average signal by visual method, and obtained the reference lookup Table. In addition, latencies and amplitudes were measured manually by two audiologists and by this technique, automatically. Using the information of reference lookup Table, periodic limitation time was set for each response and each epoch, then we used cross-correlation between grand average signal of response and response signal of each subject. In this 
step, the response signal shifted to grand average signal and produced template signal.

Coefficient correlation between template signal and stimuli signal was then applied to minimize the effect of artifacts. Finally, using earlier described periodic limitation and using pre-processing technique, all response signals of each subject were marked automatically. The values of latencies and amplitude were tabulated in different lookup Table for each stimulus and each subject.

We set individual variance for marking peaks, due to brainstem jittering in this automatic method, which means that the tolerance of each epoch was $\pm 2 \mathrm{~ms}$. ICC analyses were performed utilizing the non-normalized latencies on 4 gatherings of peaks, onset peaks 1 and 2, major peaks $3,4,6,7,9,10,12$, and 13 , minor peaks $5,8,11$, and 14 , and end-point peaks 15 and 16. A $3 \mathrm{x}$ $\mathrm{K}$ repeated measures ICC (where 3 is the quantity of stimulus conditions and $\mathrm{K}$ is the quantity of peaks) was led on each gathering. For gatherings of peaks which the stimulus $\times$ peak connection was critical, repeated measures ICC and Paired t test were performed to examine contrasts between stimulus latency.

\section{Frequency domain measures}

A Fast Fourier Transform (FFT) analysis was used for evaluating the spectral domain of response. We selected $18-58 \mathrm{~ms}$ of formant transition response time and $2000 \mathrm{~Hz}$ range of frequency. The average response was calculated in $50 \mathrm{~Hz}$ wide bins surrounding F0 and next 10 harmonics. The range of $400-720 \mathrm{~Hz}$ referring to F0 frequency was chosen and then all 10 harmonic peaks were marked for each stimulus by MATLAB software. Repeated measures ICC and Paired $t$ test were used for evaluating the significance of frequency bins for each stimulus.

\section{Results}

We compared automatic and manual methods and then compared individual differences in Persian speakers and English speakers. The automatic algorithm could correctly detect the locations of each peak in ABR signals. Additionally, the performance of this objective method is estimated $90 \%$ for all peaks, but overlooking peak 16 of $/ \mathrm{da} /$ response, the performance rate is $95 \%$ and further details are presented in Tables 2, 3, and 4. The mean score and standard deviation of the non-normalized latencies for 16 peaks picked for each stimulus condition by automatic and manual methods are described in Table $5,6,7$. The response waveform of the first $70 \mathrm{~ms}$ of transition portion, were used for comparing objective and subjective method in Persian speakers. Figures 3, 3a, and $3 \mathrm{~b}$ illustrate automatic method, and Figures 4, 4a, and $4 \mathrm{~b}$ illustrate manual method.

The within-subject main effect of stimulus $\left(\mathrm{F}_{2.52}=6.888\right.$, $\mathrm{P}=0.002)$ was significant and stimulus $\mathrm{X}$ peak interaction $\left(\mathrm{F}_{2,53}=0.863, \mathrm{P}=0.428\right)$ for the end point was nonsignificant. Also, English speakers had the same significant result for within-subject main effect of stimulus. In onset peaks 1 and 2, no significance within-subject main effect of stimulus $\left(\mathrm{F}_{1.45,37.54}=2.144, \mathrm{P}=0.147\right)$ was found, and stimulus $\mathrm{X}$ peak interaction $\left(\mathrm{F}_{2,52}=2.339, \mathrm{P}=0.107\right)$ was non-significant. Same result was obtained for English speakers. Also minor peaks have greater betweenstimulus latency differences than major peaks and their latency time is longer than major peaks. The same is true in English speakers.

\section{Frequency domain}

For analysis of transient portion in frequency domain, a range of 18-58 ms transition part was chosen. Grand averaged signal was plotted. Significant and non-significant differences were seen in this range for each stimulus. Figure 5 shows grand averaged signal and the next 10 harmonics marked by the manual method [3]. Figures $5,5 \mathrm{a}, 5 \mathrm{~b}$ show peak detection in 10 harmonics waveform for each stimulus by the automatic method. There is no significance between stim $\mathrm{X}$ peak interaction and the main within-subject effect of stimulus for each bins, because $\mathrm{P}$ values are greater than 0.05 .

The results were the same for English speakers but the results of the Paired t test for follow-up scores between these two groups were different for each harmonic. The follow-up Paired t tests for Farsi speakers harmonic were performed to assess between-stimulus

Table 1. Three Stimulus in Different Formants

\begin{tabular}{cccc}
\hline Name of Stimulus & /da/ & /ba/ & /ga/ \\
\hline Formant & F2 and F3 falling & F2 and F3 rising & FA and F3 falling \\
Range & $1700-2580 \mathrm{~Hz}$ & $900-2400 \mathrm{~Hz}$ & $3000-3100 \mathrm{~Hz}$ \\
\hline
\end{tabular}




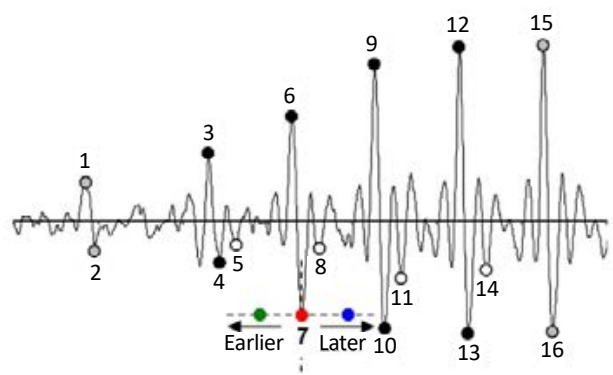

A

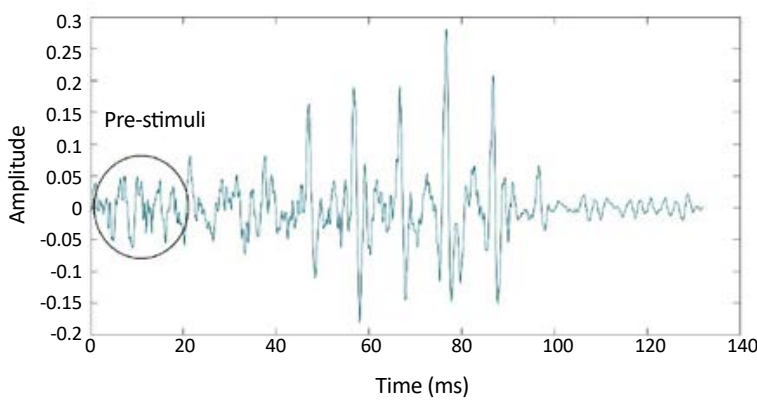

C
B

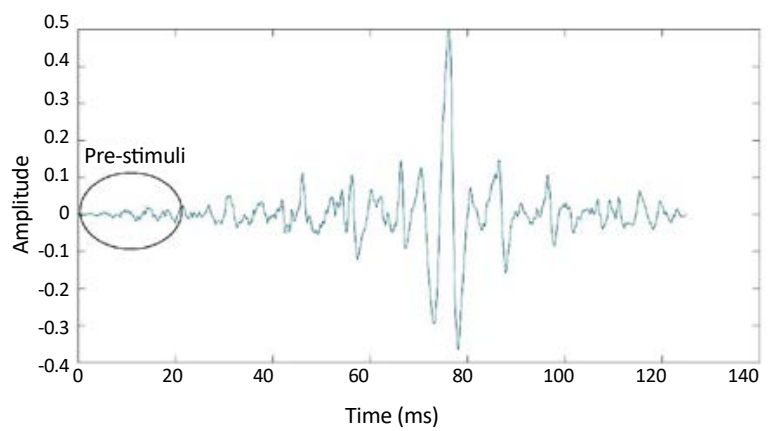

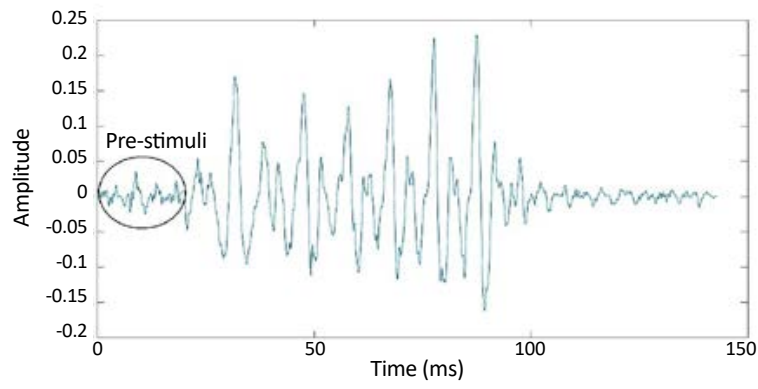

Figure 2. All 16 peaks for each stimuli in manual (visual) method [2]

Ilranian Rehabilitation Journa

A: Schematic of automatic / ba/ Grand average; B: Schematic of automatic /ga/ Grand average; C: Schematic of automatic/ da/ grand average

differences. The results are displayed in Table 8. This study revealed that in harmonics $1,3,6,9,10$, each stimulus is not significant. In harmonic 2 , only /da/ and /ba/ are significant. In harmonic 4, two pairs of stimuli / $\mathrm{da} /+/ \mathrm{ga} /$ and $/ \mathrm{ba} /+/ \mathrm{ga} /$ are significant. In harmonic 5, all stimuli are significant. In harmonic 7 only /ba/ and /ga/ are significant, and in harmonic 8, two pairs of stimuli / $\mathrm{ba} /+/ \mathrm{ga} /$ and $/ \mathrm{da} /+/ \mathrm{ga} /$ are significant.

\section{Discussion}

The purpose of this study was to describe new simple automatic peak detection for extracting features of cABR. In this study, a cross-correlation between grand average signal of all subject response and response signal was used for shifting signals of each subject across the grand average signal and producing template sig-

Table 2. Comparing manual and automatic method in all peaks

\begin{tabular}{cccc}
\hline \multicolumn{5}{c}{ ICC } & \\
\hline \multicolumn{5}{c}{ Stimulus } \\
\hline General & $/ \mathrm{da} /$ & $/ \mathrm{ba} /$ & $/ \mathrm{ga} /$ \\
0.998 & 0.995 & 0.999 & 0.999 \\
\hline
\end{tabular}

Ilranian Rehabilitation Journal 
A

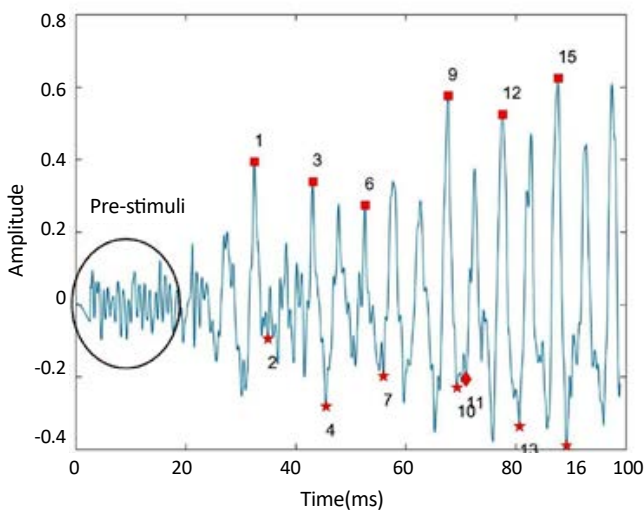

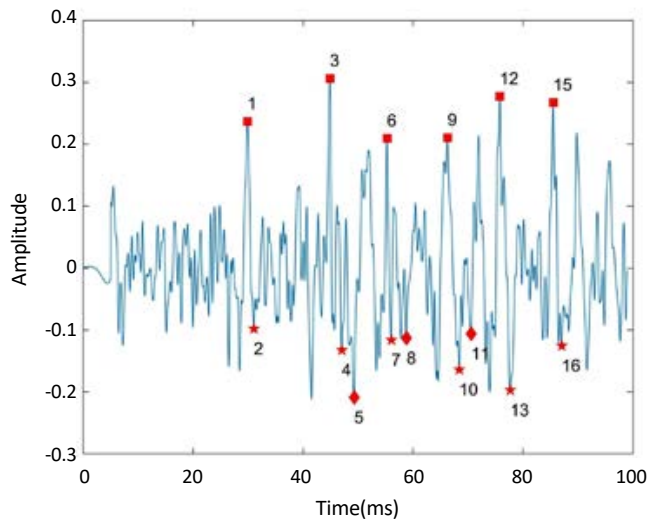

C

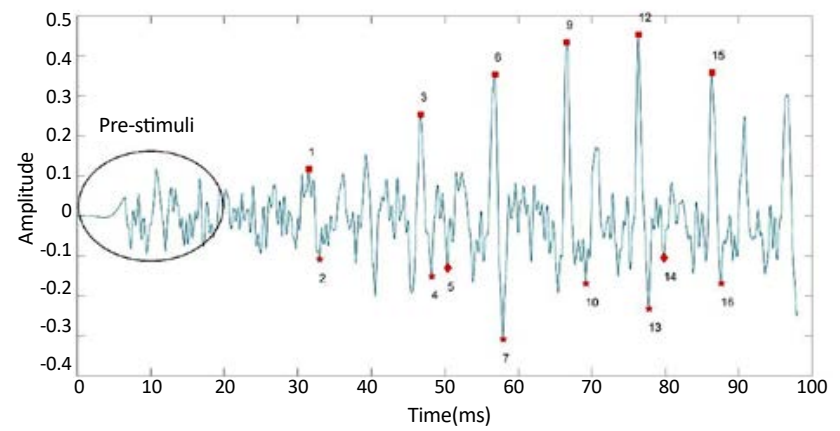

IIranian Rehabilitation Journal

Figure 3. a. Peak detection of /da/ in automated ABR; b: Peak detection of / ba/ in automated ABR

nal. With the use of limitation periodic time, this automatic quality evaluation method was compared to a subjective evaluation by two experts of audiology and electro physiology. The results indicated that automatic method presents a $90 \%$ correlation coefficients averaged with the visual assessment. It means that our first hypothesis (automatic algorithm used for detecting peaks) is similar to visual analysis.
The figures show an important bias among experts in the subjective method, which means that visual judgment is not really exhaustive $[15,16,18]$. Comparing the subjective and objective method showed that automatic methods are uniform, worldwide, useful, and eliminate human bias. There is no limitation for the number of data [11]. Therefore, automatic algorithm could extract latency time in high accuracy and improve fractional milliseconds.

Table 3. Performance rate of comparing manual and automatic method in each peak separately

\begin{tabular}{ccccccccccccccccc}
\hline Peak & $\mathbf{1}$ & $\mathbf{2}$ & $\mathbf{3}$ & $\mathbf{4}$ & $\mathbf{5}$ & $\mathbf{6}$ & $\mathbf{7}$ & $\mathbf{8}$ & $\mathbf{9}$ & $\mathbf{1 0}$ & $\mathbf{1 1}$ & $\mathbf{1 2}$ & $\mathbf{1 3}$ & $\mathbf{1 4}$ & $\mathbf{1 5}$ & $\mathbf{1 6}$ \\
\hline ICC & 0.96 & 0.98 & 0.982 & 0.959 & 0.888 & 0.989 & 0.969 & 0.885 & 0.967 & 0.848 & 0.99 & 0.908 & 0.879 & 0.917 & 0.948 & 0.64
\end{tabular}

Table 4. Comparing manual and automatic methods in each peak for each stimulus

\begin{tabular}{|c|c|c|c|c|c|c|c|c|c|c|c|c|c|c|c|c|c|}
\hline & & P1 & P2 & P3 & P4 & P5 & P6 & P7 & P8 & P9 & p10 & p11 & p12 & p13 & P14 & P15 & p16 \\
\hline da & 1 & 0.986 & 0.987 & 0.981 & 0.968 & 0.670 & 0.997 & 0.995 & 0.829 & 0.834 & 0.776 & 0.998 & 0.796 & 0.573 & 0.945 & 0.866 & $0.131-$ \\
\hline ba & C & 0.994 & 0.983 & 0.915 & 0.941 & 0.871 & 0.956 & 0.950 & 0.671 & 0.985 & 0.797 & 0.655 & 0.838 & 0.834 & 0.761 & 0.950 & 0.913 \\
\hline ga & C & 0.959 & 0.929 & 0.961 & 0.926 & 0.948 & 0.965 & 0.895 & 0.975 & 0.854 & 0.979 & 0.862 & 0.975 & 0.982 & 0.983 & 0.991 & 0.797 \\
\hline
\end{tabular}



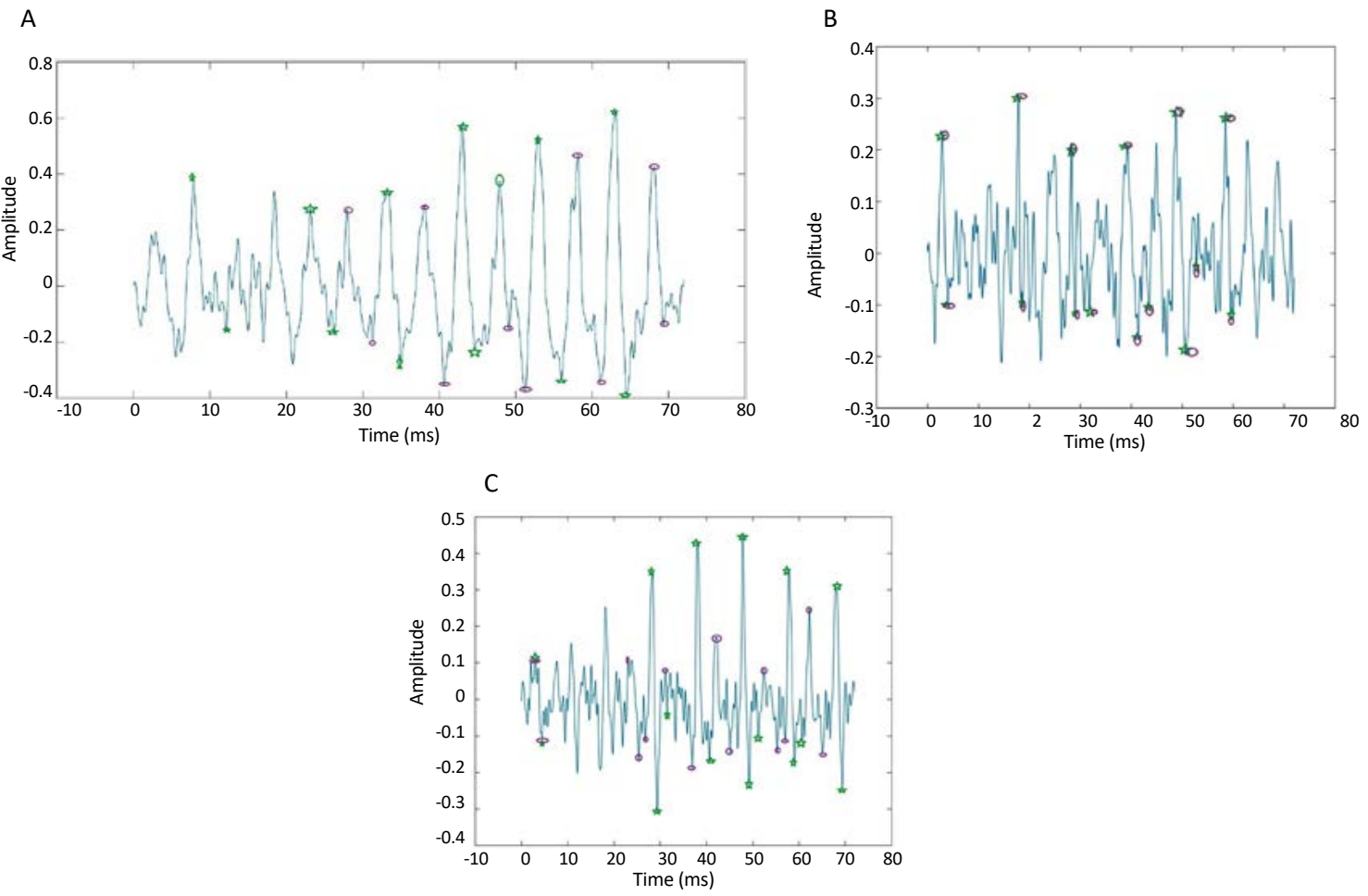

Figure 4. a: Peak detection of /da/ in manual; b: Peak detection of / ba/ in automated ABR

IIranian Rehabilitation Wournal

Table 5. Mean \pm SD for / da/ responses

\begin{tabular}{cccc}
\hline & & [da] & \\
\cline { 2 - 4 } Peak & & Mean \pm SD & Expert 2 \\
\cline { 2 - 4 } & Automatic Algorithm & Expert 1 & $8.585185 \pm 1.103963$ \\
\hline 1 & $8.511111 \pm 1.041443$ & $8.584444 \pm 1.103834$ & $10.90296 \pm 0.972193$ \\
\hline 2 & $10.91889 \pm 0.962118$ & $10.84333 \pm 1.06689$ & $24.54148 \pm 0.9664$ \\
\hline 3 & $24.59667 \pm 0.937423$ & $24.36926 \pm 1.020086$ & $27.08 \pm 1.149394$ \\
\hline 5 & $27.11333 \pm 1.072646$ & $27.20407 \pm 1.172575$ & $29.38815 \pm 0.788421$ \\
\hline 6 & $29.30741 \pm 0.733782$ & $30.3737 \pm 0.432552$ & $34.11926 \pm 2.11143$ \\
\hline 7 & $34.15074 \pm 2.108849$ & $33.9563 \pm 2.247079$ & $36.79593 \pm 1.044264$ \\
\hline 9 & $36.84148 \pm 1.021841$ & $36.82259 \pm 1.034425$ & $38.25815 \pm 0.708096$ \\
\hline 10 & $38.56704 \pm 0.567984$ & $38.18333 \pm 0.54089$ & $44.01222 \pm 1.422969$ \\
\hline 11 & $44.31296 \pm 1.224537$ & $43.94704 \pm 1.270902$ & $45.85519 \pm 1.145645$ \\
\hline 12 & $45.77259 \pm 0.783079$ & $45.95407 \pm 0.902383$ & $44.92481 \pm 4.612287$ \\
\hline 13 & $45.23407 \pm 4.588122$ & $44.85 \pm 4.563259$ & $53.8363 \pm 1.382175$ \\
\hline 14 & $54.50037 \pm 1.170662$ & $54.06519 \pm 1.16963$ & $56.30037 \pm 1.097986$ \\
\hline 15 & $56.68556 \pm 1.28916$ & $56.5 \pm 0.833528$ & $58.18926 \pm 0.909818$ \\
\hline & $58.30815 \pm 0.820296$ & $58.0637 \pm 0.760345$ & $64.03333 \pm 1.429389$ \\
\hline
\end{tabular}

Iranian Rehabilitation Journal 
Table 6. Mean $\pm S D$ of Persian speakers by automatic and manual method

\begin{tabular}{|c|c|c|c|}
\hline \multirow{3}{*}{ Peak } & \multicolumn{3}{|c|}{ [ba] } \\
\hline & \multicolumn{3}{|c|}{ Mean $\pm S D$} \\
\hline & Automatic Algorithm & Expert 1 & Expert 2 \\
\hline 1 & $8.4767 \pm 0.77408$ & $8.4000 \pm 0.78356$ & $8.4537 \pm 0.76682$ \\
\hline 2 & $10.7852 \pm 0.49453$ & $10.7459 \pm 0.50217$ & $10.903 \pm 0.45681$ \\
\hline 3 & $22.2193 \pm 0.52022$ & $22.0122 \pm 0.58136$ & $22.3615 \pm 0.45794$ \\
\hline 4 & $25.6741 \pm 1.18459$ & $25.74 \pm 1.00944$ & $25.7289 \pm 1.13961$ \\
\hline 5 & $28.548 \pm 0.77462$ & $28.0963 \pm 0.56684$ & $28.2541 \pm 0.8391$ \\
\hline 6 & $32.0037 \pm 1.0261$ & $31.8578 \pm 1.3106$ & $32.093 \pm 0.91653$ \\
\hline 7 & $35.2763 \pm 1.25693$ & $35.1533 \pm 1.21899$ & $35.3252 \pm 1.17312$ \\
\hline 8 & $37.6678 \pm 0.65813$ & $37.7711 \pm 0.82257$ & $37.8511 \pm 0.85382$ \\
\hline 9 & $41.0804 \pm 1.14280$ & $40.7096 \pm 0.99273$ & $40.8078 \pm 0.98878$ \\
\hline 10 & $40.8078 \pm 0.98878$ & $45.4019 \pm 1.24985$ & $45.7044 \pm 0.94282$ \\
\hline 11 & $47.4956 \pm 0.81141$ & $46.9970 \pm 0.88363$ & $47.9441 \pm 0.90349$ \\
\hline 12 & $52.6763 \pm 1.38569$ & $52.6052 \pm 1.33711$ & $53.0652 \pm 1.25570$ \\
\hline 13 & $54.8933 \pm 1.54071$ & $54.5970 \pm 1.29167$ & $54.7874 \pm 1.10067$ \\
\hline 14 & $57.3056 \pm 1.10869$ & $57.1537 \pm 0.94326$ & $57.4200 \pm 0.70161$ \\
\hline 15 & $62.9004 \pm 1.35706$ & $62.9885 \pm 1.41514$ & $62.9811 \pm 1.50291$ \\
\hline 16 & $64.5304 \pm 1.62949$ & $64.7663 \pm 1.51650$ & $64.8900 \pm 1.60562$ \\
\hline
\end{tabular}

The second purpose of this study was to compare the relationship between the automatic and manual method. Each method showed that brainstem response among manufactured voiced stop consonants /ga/, /da/, and $/ \mathrm{ba} /$ recurrence advances are extraordinary only in F2 and F3. Since the scope of F2 and F3 are over the phase-locking capacities of the brainstem recurrence, they would be shown as latency contrasts among responses. It implies responses to /ga/ and would have the most punctual latencies. As it contains the most astounding F2 and F3 frequencies, responses to /ba/ would have the most recent latencies because of having the least F2 and F3 frequencies, and responses to [da] would have middle latency responses [2].

Skoe et al. used the fast Fourier analysis and mentioned that $\mathrm{F} 2$ and $\mathrm{F} 3$ recurrence ranges are higher than the phase-locking of the brainstem response, consequently recurrence contrasts are characterized as latency differences due to responses. Harmonics figure illustrated that the most similarities are between /da/ and /ga/ neural encoding, and the most dissimilarities are between $/ \mathrm{ba} /$ and $/ \mathrm{ga} /$ of their neural encoding. This explains that the formant frequency of each stimulus causes dissimilarity or similarity in auditory response [2]. The same results were obtained for our third hypothesis that automatic algorithm could be used as a co-observer in clinics. The latency time of onset response of $/ \mathrm{ba} /, / \mathrm{da} /$ and $/ \mathrm{ga} /$ for Persian speakers, are later than English speakers. We hypothesized that these differences of latency time between Persian speakers and English speakers are related to differences in age, language training, and plasticity of brainstem in these two groups.

\section{Language experience}

Language experience plays a critical role in the development of neural encoding in auditory system at the place of cortical and subcortical levels [20]. Evidence has shown that when native people listen to their native 
Table 7. Mean $\pm S D$ of Persian speakers by automatic and manual method

\begin{tabular}{|c|c|c|c|}
\hline \multirow{3}{*}{ Peak } & \multicolumn{3}{|c|}{ [ga] } \\
\hline & \multicolumn{3}{|c|}{ Mean \pm SD } \\
\hline & Automatic Algorithm & Expert 1 & Expert 2 \\
\hline 1 & $8.4167 \pm 0.34199$ & $8.3859 \pm 0.39048$ & $8.4144 \pm 0.32803$ \\
\hline 2 & $10.3819 \pm 0.51248$ & $10.3619 \pm 0.50739$ & $10.4574 \pm 0.39529$ \\
\hline 3 & $23.7807 \pm 0.99680$ & $23.9037 \pm 0.94578$ & $23.6722 \pm 0.90747$ \\
\hline 4 & $26.2493 \pm 1.15239$ & $26.2352 \pm 1.13071$ & $26.2741 \pm 1.05539$ \\
\hline 5 & $28.4259 \pm 0.35469$ & $28.3856 \pm 0.31075$ & $28.5311 \pm 0.33397$ \\
\hline 6 & $33.2222 \pm 1.28941$ & $33.3193 \pm 1.11055$ & $33.4252 \pm 1.11446$ \\
\hline 7 & $35.6822 \pm 0.80493$ & $35.8822 \pm 0.79950$ & $35.5526 \pm 0.54696$ \\
\hline 8 & $37.3600 \pm 0.77473$ & $37.4215 \pm 0.73529$ & $37.3996 \pm 0.77807$ \\
\hline 9 & $43.8144 \pm 0.89528$ & $43.9322 \pm 0.83028$ & $44.2015 \pm 0.60570$ \\
\hline 10 & $45.9748 \pm 0.84481$ & $46.0070 \pm 0.82488$ & $45.9041 \pm 0.78112$ \\
\hline 11 & $48.2470 \pm 0.61401$ & $48.5037 \pm 0.66133$ & $48.5744 \pm 0.63169$ \\
\hline 12 & $53.3585 \pm 1.31262$ & $53.3104 \pm 1.30699$ & $53.5463 \pm 1.33024$ \\
\hline 13 & $55.3722 \pm 0.87446$ & $55.3563 \pm 0.86687$ & $55.5893 \pm 0.86692$ \\
\hline 14 & $58.3315 \pm 0.53064$ & $58.3367 \pm 0.49425$ & $58.4974 \pm 0.55796$ \\
\hline 15 & $63.5233 \pm 1.16696$ & $63.4826 \pm 1.16705$ & $63.7111 \pm 1.15688$ \\
\hline 16 & $65.7252 \pm 1.40925$ & $65.6137 \pm 1.32373$ & $66.6870 \pm 1.57679$ \\
\hline
\end{tabular}

A

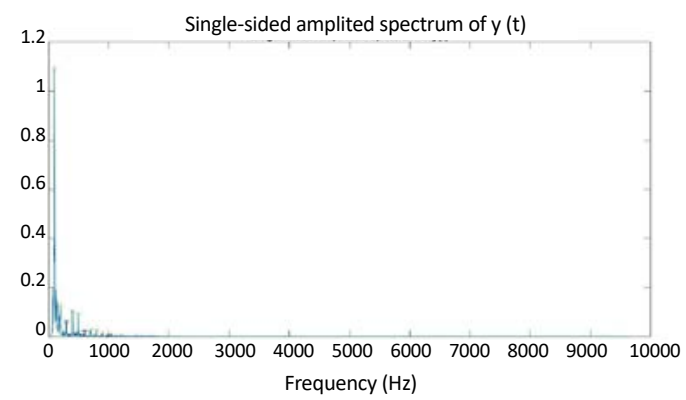

Single-sided amplited spectrum of $\mathrm{y}(\mathrm{t})$

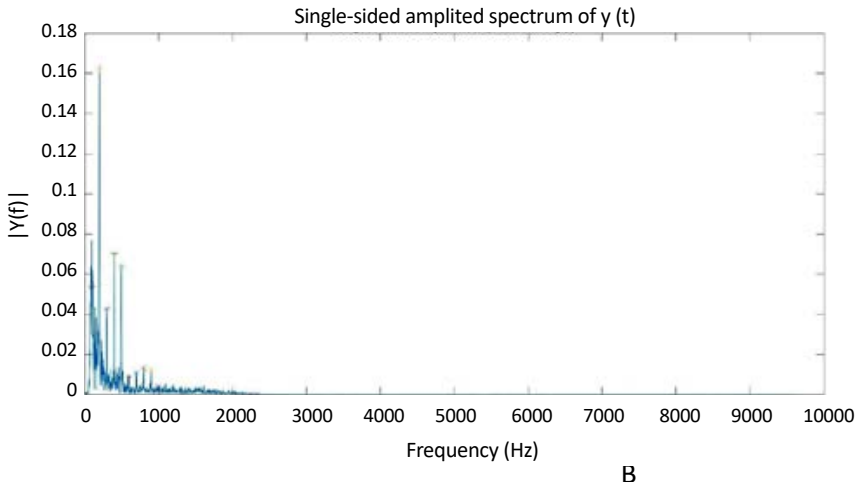

B

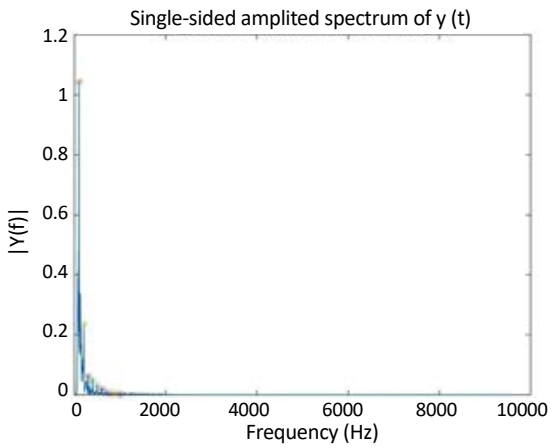

Figure 5. Automatic/da/ FFT peak detection in 10 harmonics

Ilranian Rehabilitation Journal

a: Automatic / ba/ FFT peak detection in 10 harmonics; b: Automatic /ga/ FFT peak detection in 10 harmonics 
Table 8. Follow-up paired t test results for harmonics in Persian speakers

\begin{tabular}{cccccc}
\hline Stimuluses Harmonics & Pair & Mean & SD & Paired t-Test & P \\
\hline ba_H4 - da_H4 & 1 & 0.0008358 & 0.0078506 & 0.553 & 0.585 \\
ba_H4 -ga_H4 & 2 & 0.0054945 & 0.0072078 & 3.961 & 0.001 \\
da_H4 - ga_H4 & 3 & 0.0046587 & 0.0071720 & 3.375 & 0.002 \\
ba_H5 - da_H5 & 4 & 0.0016954 & 0.0049252 & 1.789 & 0.085 \\
ba_H5 - ga_H5 & 5 & 0.0047706 & 0.0045544 & 5.443 & 0.000 \\
\hline da_H5 - ga_H5 & 6 & 0.0030753 & 0.0059645 & 2.679 & 0.013 \\
\hline ba_H7 - da_H7 & 7 & 0.0003285 & 0.0023994 & 0.711 & 0.483 \\
ba_H7 - ga_H7 & 8 & 0.0010368 & 0.0021118 & 2.551 & 0.017 \\
da_H7 - ga_H7 & 9 & 0.0007083 & 0.0021384 & 1.721 & 0.097 \\
\hline ba_H8 - da_H8 & 10 & 0.0003592 & 0.0018044 & 1.035 & 0.310 \\
\hline ba_H8 - ga_H8 & 11 & 0.0009422 & 0.0016689 & 2.934 & 0.007 \\
\hline da_H8 - ga_H8 & 12 & 0.0005829 & 0.0014867 & 2.037 & 0.052 \\
\hline
\end{tabular}

stimuli, the F0 of brainstem response are larger than the non-native speakers [20-22].

According to their early learning, the formation and functional properties of neural organization means high skills in detecting and predicting native language [20]. Neural representation of pitch reveals that language experience can affect the behavior of action potential and sound processing in brainstem and cortex $[22,23]$. Cellular adaptations lead to plasticity in brainstem and cortex, which implies vast somatic synapses, quick discharge time course, quick AMPA receptor energy, plasticity prompts brief synaptic responses that advances insignificant worldly summation, balanced flagging, short-latency spikes, and a short hard-headed period [23, 24].

Age

Maturation influences the transmission time. It influences the peripheral auditory pathway maturity amid the initial 2 months of life, while the central transmission time abbreviates up to the age of 5 to 8 years. The III-II and V-IV inter peak latencies demonstrated maturational changes like those of V-I IPLs, interestingly, II-I and IVIII indicated little changes. An unmistakable increment of the amplitude of peak $\mathrm{V}$ up to age of 4 and a consequent decreasing tendency was observed. In this study, Persian speakers were between 22 and 28 years old, and English speakers between 8 and 12 years. This age dif- ference leads to differences in morphology of V-I peaks and amplitude of response [25, 26]. Results indicated that separate latency and amplitude norms for English speakers and Persian speakers have worthwhile significance to cABR measures [25].

By overviewing the result of this study, it seems that using more professional audiologists in speech ABR field could increase the precision of grand average in the visual method and facilitates comparing the methods. By reducing the time of recording, artificial noise and mental fatigue could decrease. Future studies could use Linear Discriminant Analysis (LDA) classifier for analyzing or extracting the effect of mental fatigue on latency time.

\section{Gender}

Gender is a physiological factor that can affect brainstem auditory, evoked potential responses, and latencies. Head size and Body Mass Index (BMI) in different age and genders are different; these differences could show up in latencies of peaks I-III [26].

\section{Conclusion}

The automatic algorithm could detect all 16 peaks in brainstem response signals, and extract latency time with high accuracy. There is no visual bias in this algorithm. Time limitation including individual variance 
provides higher precision for calculating latency time. The high dissimilarity between different experts and automatic algorithm is in peaks 15 and 16 , because there are offset and the endpoint of signals and downing in most artifacts.

\section{Ethical Considerations}

\section{Compliance with ethical guidelines}

Prior to study, all participants signed a written informed consent, approved by the Ethics Committee of Tehran University of Medical Sciences. They were rewarded for their participation.

Funding

This research did not receive any specific grant from funding agencies in the public, commercial, or not-forprofit sectors.

\section{Conflict of interest}

No irreconcilable interest were identified with this research. The authors certify that they have no affiliation with or involvement in any organization or entity with any financial, or non-financial interest in the subject matter or materials dismissed in this manuscript.

\section{Acknowledgments}

This study was a part of master's thesis of Negar Amirian, supported by Islamic Azad University, Central Tehran Branch. The authors gratefully acknowledge Dr. Bram Van Dun for his unforgettable support during this study and marking the peaks of brainstem response waves, Dr. Zahra Shirjiyan for her kind assistance, Dr. Mohsin Reza Heydari, Dr. Ali Akbar Tahaei, and Dr. Mohsen Ahadi for their guidance regarding neuroscience and auditory system during this study.

\section{References}

[1] Jewett DL, Williston JS. Auditory-evoked far fields averaged from the scalp of humans. Brain. 1971; 94(4):681-96 [DOI:10.1093/brain/94.4.681] [PMID]

[2] Johnson KL, Nicol T, Zecker SG, Bradlow AR, Skoe E, Kraus $\mathrm{N}$. Brainstem encoding of voiced consonant-vowel stop syllables. Clinical Neurophysiology. 2008; 119(11):2623-5. [DOI:10.1016/j.clinph.2008.07.277] [PMID]

[3] Blumstein SE, Isaacs E, Mertus J. The role of the gross spectral shape as a perceptual cue to place of articulation in ini- tial stop consonants. The Journal of the Acoustical Society of America. 1982; 72(1):43-50. [DOI:10.1121/1.388023] [PMID]

[4] Ballachanda BB, Moushegian G, Stillman RD. Adaptation of the auditory brainstem response: Effects of click intensity, polarity, and position. Journal of the American Academy of Audiology. 1992; 3(4):275-82. [PMID]

[5] Valderrama JT, de la Torre A, Alvarez I, Segura JC, Thornton ARD, Sainz M, et al. Automatic quality assessment and peak identification of auditory brainstem responses with fitted parametric peaks. Computer Methods and Programs in Biomedicine. 2014; 114(3):262-75. [DOI:10.1016/j.cmpb.2014.02.015] [PMID]

[6] Don M, Elberling C. Use of quantitative measures of auditory brainstem response peak amplitude and residual background noise in the decision to stop averaging. The Journal of the Acoustical Society of America. 1996; 99(1):491-9. [DOI:10.1121/1.414560] [PMID]

[7] Sparacino G, Milani S, Arslan E, Cobelli C. A Bayesian approach to estimate evoked potentials. Computer Methods and Programs in Biomedicine. 2002; 68(3):233-48. [DOI:10.1016/ S0169-2607(01)00175-4]

[8] Sadeghian A, Dajani HR, Chan ADC. Classification of speech-evoked brainstem responses to English vowels. Speech Communication. 2015; 68:69-84. [DOI:10.1016/j. specom.2015.01.003]

[9] Fridman J, John E, Bergelson M, Kaiser J, Baird H. Application of digital filtering and automatic peak detection to brain stem auditory evoked potential. Electroencephalography and Clinical Neurophysiology. 1982; 53(4):405-16 [DOI:10.1016/0013-4694(82)90005-0]

[10] Chan FHY, Lam FK, Poon PWF, Qiu W. Detection of brainstem auditory evoked potential by adaptive filtering. Medical and Biological Engineering and Computing. 1995; 33(1):69-75. [DOI:10.1007/BF02522949] [PMID]

[11] Grönfors T. Peak identification of auditory brainstem responses with multifilters and attributed automaton. Computer Methods and Programs in Biomedicine. 1993; 40(2):83-7. [DOI:10.1016/0169-2607(93)90002-3]

[12] Galbraith GC. Enhanced brainstem and cortical evoked response amplitudes: Single-trial covariance analysis. Perceptual and Motor Skills. 2001; 92(3):659-72. [DOI:10.2466/ pms.2001.92.3.659] [PMID]

[13] Sundaramoorthy V, Pont MJ, Degg C, Cook JA. A computerized database of 'normal'auditory brainstem responses. British Journal of Audiology. 2000; 34(3):197-201. [DOI:10.3109/03005364000000129] [PMID]

[14] Vannier E, Adam O, Motsch JF. Objective detection of brainstem auditory evoked potentials with a priori information from higher presentation levels. Artificial Intelligence in Medicine. 2002; 25(3):283-301. [DOI:10.1016/S0933-3657(02)00029-5]

[15] Weber BA, Fletcher GL. A computerized scoring procedure for auditory brainstem response audiometry. Ear and Hearing. 1980 1(5):233-6. [DOI:10.1097/00003446-198009000-00001] [PMID]

[16] Arnold SA. Objective versus visual detection of the auditory brain stem response. Ear and Hearing. 1985; 6(3):144-50 [DOI:10.1097/00003446-198505000-00004] [PMID]

[17] Kakiashvili T, Koczkodaj WW, Woodbury-Smith M. Improving the medical scale predictability by the pairwise 
comparisons method: Evidence from a clinical data study. Computer Methods and Programs in Biomedicine. 2012 105(3):210-6. [DOI:10.1016/j.cmpb.2011.09.011] [PMID]

[18] Jafarpisheh AS, Jafari AH, Abolhassani M, Farhadi M, Sadjedi H, Pourbakht A, et al. Nonlinear feature extraction for objective classification of complex auditory brainstem responses to diotic perceptually critical consonant-vowel syllables. Auris Nasus Larynx. 2016; 43(1):37-44. [DOI:10.1016/j. anl.2015.06.003] [PMID]

[19] McMath RC. Engineering the New South: Georgia Tech, 1885-1985. Athens: University of Georgia Press; 1985.

[20] Skoe E, Kraus N. Auditory brainstem response to complex sounds: A tutorial. Ear and Hearing. 2010; 31(3):302-4. [DOI:10.1097/AUD.0b013e3181cdb272] [PMID] [PMCID]

[21] Galbraith GC, Bhuta SM, Choate AK, Kitahara JM, Mullen TA. Brain stem frequency-following response to dichotic vowels during attention. Neuroreport. 1998; 9(8):1889-93. [DOI:10.1097/00001756-199806010-00041] [PMID]

[22] Krishnan A, Gandour JT, Bidelman GM. Experience-dependent plasticity in pitch encoding: From brainstem to auditory cortex. Neuroreport. 2012; 23(8):498-502. [DOI:10.1097/ WNR.0b013e328353764d] [PMID] [PMCID]

[23] Zounopoulos T, Kraus N. Learning to encode timing: Mechanisms of plasticity in the auditory brainstem. Neuron. 2009; 62(4):463-9. [DOI:10.1016/j.neuron.2009.05.002] [PMID] [PMCID]

[24] Trussell LO. Synaptic mechanisms for coding timing in auditory neurons. Annual Review of Physiology. 1999; 61(1):477-96. [DOI:10.1146/annurev.physiol.61.1.477] [PMID]

[25] Mochizuki Y, Go T, Ohkubo H, Tatara T, Motomura T. Developmental changes of Brainstem Auditory Evoked Potentials (BAEPs) in normal human subjects from infants to young adults. Brain and Development. 1982; 4(2):127-36. [DOI:10.1016/S0387-7604(82)80006-5]

[26] Stockard JE. Brainstem auditory-evoked responses: Normal variation as a function of stimulus and subject characteristics. Archives of Neurology. 1979; 36(13):823-31. [DOI:10.1001/ archneur.1979.00500490037006] [PMID] 
